\title{
SOCIOLOGIE Sociologie du travail
}

DU TRAVAIL

Vol. 57 - $n^{\circ} 1$ | Janvier-Mars 2015

Participer. Pour quoi faire?

\section{Troubles en psychiatrie. Enquête dans une unité pour adolescents, I. Coutant}

La Dispute, Paris (2012). 216 p.

Nicolas Henckes

\section{OpenEdition}

Journals

Édition électronique

URL : https://journals.openedition.org/sdt/1871

DOI : $10.4000 /$ sdt. 1871

ISSN : $1777-5701$

Éditeur

Association pour le développement de la sociologie du travail

Édition imprimée

Date de publication : 1 mars 2015

Pagination : 143-144

ISSN : 0038-0296

Ce document vous est offert par Centre national de la recherche scientifique (CNRS)

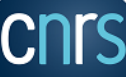

Référence électronique

Nicolas Henckes, «Troubles en psychiatrie. Enquête dans une unité pour adolescents, I. Coutant 》, Sociologie du travail [En ligne], Vol. 57 - n 1 | Janvier-Mars 2015, mis en ligne le 27 janvier 2015, consulté le 19 janvier 2022. URL : http://journals.openedition.org/sdt/1871 ; DOI : https://doi.org/ $10.4000 /$ sdt. 1871

Ce document a été généré automatiquement le 19 janvier 2022.

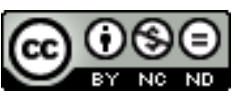

Sociologie du travail is licensed under a Creative Commons Attribution-NonCommercial-NoDerivatives 4.0 International License. 


\title{
Troubles en psychiatrie. Enquête dans une unité pour adolescents, I. Coutant
}

La Dispute, Paris (2012). 216 p.

\author{
Nicolas Henckes
}

\section{RÉFÉRENCE}

Isabelle Coutant, Troubles en psychiatrie. Enquête dans une unité pour adolescents, La Dispute, Paris, 2012, 216 p.

1 L'ouvrage d'Isabelle Coutant ressortit à un genre, la monographie d'institution psychiatrique, qui non seulement a donné à la sociologie un certain nombre de ses classiques, mais est de surcroît aujourd'hui encore régulièrement enrichi de travaux originaux. Dans ces conditions, quatre tests peuvent être mobilisés pour juger l'intérêt de la contribution d'I. Coutant: la pertinence de l'institution choisie pour terrain, l'originalité du questionnement mis en œuvre, la richesse des observations au service de ce questionnement et, finalement et bien entendu, la qualité des analyses. Disons d'emblée que Troubles en psychiatrie paraît passer ces différents tests avec un succès inégal.

2 L'ouvrage renferme d'excellents arguments pour aborder les deux premiers. I. Coutant s'est installée dans une unité hospitalière accueillant des adolescents souffrant de troubles des conduites et située dans une banlieue défavorisée. Parce que les problèmes de ces jeunes sont mal définis et peu spectaculaires, croisant le social et le psychiatrique, le travail auprès d'eux offre peu de gratifications aux professionnels des mondes psy qui sont par ailleurs peu enclins à s'installer dans une zone mal desservie de l'espace social. L'intérêt de l'enquête sociologique sur ces pratiques peu valorisées, si ce n'est invisibles, serait ainsi amplement justifié par sa seule dimension proprement documentaire. Il est renforcé par le fait que l'unité en question était par ailleurs, au 
moment où I. Coutant a réalisé ses observations, animée par un petit noyau de cliniciens adhérant encore aux idéaux du mouvement réformateur de la psychiatrie des années 1960 et 1970 et qui continuaient à organiser le travail de leur équipe selon les principes de la "psychothérapie institutionnelle » hérités de cette période. Ces idéaux étaient cependant mis en difficulté par le manque de moyens et la dégradation des conditions de travail, la précarisation du personnel soignant, les difficultés du travail clinique avec une population cumulant les difficultés, mais aussi la montée au sein de la psychiatrie, et notamment au sein de l'équipe soignante elle-même, d'autres conceptions du travail psychiatrique. L'institution était ainsi traversée par une série de tensions qui en font un analyseur particulièrement riche de la psychiatrie d'aujourd'hui et des façons dont elle accomplit ses fonctions auprès d'une frange importante de ses clientèles défavorisées.

3 I. Coutant aborde ce terrain avec un questionnement développé sur ses terrains antérieurs: comment fonctionnent les institutions d'encadrement de la jeunesse populaire? Ce questionnement la conduit à croiser l'analyse du travail psychiatrique avec une réflexion sur les rapports de classe tels qu'ils peuvent s'observer entre les différents acteurs du service : entre patients et soignants autant qu'à l'intérieur de chaque groupe, au sein du groupe des patients et surtout de celui des soignants, voire même au sein des différentes catégories professionnelles composant ce dernier, et notamment du corps médical. Jusqu'à quel point le travail de l'institution est-il façonné par ces rapports sociaux? Quelles sont les ressources différentielles dont disposent les adolescents et leurs familles pour faire face aux pratiques institutionnelles? On ne saurait trop souligner la richesse de ce questionnement qui ouvre une série de perspectives stimulantes pour l'ethnographie de la psychiatrie. Alors que celle-ci tend à trop souvent ramener les usagers de la psychiatrie à leur condition de patients, la prise en compte des trajectoires sociales ouvre en effet un espace analytique pour rendre compte de l'impact différentiel des pratiques sur les individus et aboutir ainsi à une vision moins monolithique de l'institution psychiatrique.

4 Le programme est stimulant, on le voit. Sa mise en œuvre paraît cependant en deçà des attentes qu'il pourrait faire naître, en raison d'une série de choix concernant à la fois, et de façon probablement indissociable, l'enquête et l'écriture. I. Coutant concentre ses analyses sur le travail de l'institution et si elle indique avoir elle-même circulé en dehors de cette dernière pour rencontrer certains patients et leurs familles, les données qu'elle a pu recueillir par ce biais n'organisent pas l'écriture. On pourrait regretter qu'elle n'ait pas mis davantage à profit sa connaissance du terrain pour par exemple restituer le rôle de l'unité dans l'écologie locale des institutions sociales autant que dans le façonnement des trajectoires individuelles, mais au moins ce choix est-il assumé et l'analyse est, à l'intérieur de ce cadre, conduite avec cohérence au fil de l'ouvrage. Après un premier chapitre proposant une présentation de l'unité et de son personnel, les chapitres suivants découpent ainsi le travail des acteurs en suivant pratiquement chronologiquement le déroulement des prises en charge : qualification des problèmes des jeunes ; organisation, maintien et imposition de la discipline institutionnelle; travail de construction de soi; travail d'inscription dans un milieu familial. L'ensemble est clairement écrit et les différents chapitres contiennent de nombreuses observations pertinentes, recoupant souvent la littérature existante sans cependant la citer toujours - et apportant parfois des notations originales. 
5 La difficulté vient de ce que l'institution psychiatrique ne sert pas seulement de cadre aux observations, mais aussi au positionnement de la sociologue. Tout se passe en effet comme si I. Coutant développait ses analyses dans un espace qui lui était assigné par la psychiatrie, sans essayer elle-même de s'en émanciper. Ainsi le développement est-il construit à partir de récits de «cas » qui redoublent ou prolongent les cas cliniques, mobilisant par ailleurs le matériau issu des dossiers médicaux en le traitant sur le même plan que celui obtenu par les observations, celles-ci s'ajoutant à celles des cliniciens et soignants. I. Coutant commente plus qu'elle ne discute les catégories et savoirs professionnels et si elle examine les ressorts des décisions, c'est uniquement dans la mesure où le raisonnement clinique lui paraît d'une certaine façon contrecarré par d'autres schèmes de causalité, et particulièrement des rapports de classe. Plus généralement, elle concentre ses observations sur les moments de discussion formelle auxquels elle a pu assister, occultant le travail informel mené tout au long des journées. Le lecteur ainsi mis face à deux déterminismes qui n'interagissent pas, psychiatrique et sociologique, est finalement conduit à s'interroger sur l'intérêt d'une explication sociologique qui paraît souvent s'arrêter au seuil de ce qui pourrait être analysé.

\section{AUTEURS}

\section{NICOLAS HENCKES}

Centre de recherche médecine, sciences, santé, santé mentale et société (CERMES3), UMR CNRS, INSERM, EHESS et Université Paris-V, Campus CNRS, 7, rue Guy Môquet, 94801 Villejuif Cedex, France

henckes[at]vjf.cnrs.fr 